\title{
Introduction to the second issue on machine translation for low-resource languages
}

\author{
Chao-Hong Liu ${ }^{1} \cdot$ Alina Karakanta $^{2} \cdot$ Audrey N. Tong $^{3} \cdot$ Oleg Aulov $^{3}$. \\ Ian M. Soboroff ${ }^{3} \cdot$ Jonathan Washington ${ }^{4} \cdot$ Xiaobing Zhao $^{5}$
}

Accepted: 29 April 2021 / Published online: 12 May 2021

(c) The Author(s), under exclusive licence to Springer Nature B.V. 2021

In this second issue of the special topic on machine translation (MT) for lowresource languages, we include four papers that introduce knowledge in addition to those automatically learned from parallel corpora.

The paper by Armengol-Estapé and Costa-Jussà is a survey on the use of semantic and linguistic information in neural MT (NMT). It reviews methods making use of these features in NMT models and explores the use of linked data from BabelNet and Babelfy. Preliminary experiments show improved MT performance on both high (German-to-English) and low (English-to-Nepali) resource language pairs. It would be interesting to see more research efforts in this direction for other languages.

Chao-Hong Liu

ch.liu@acm.org

Alina Karakanta akarakanta@fbk.eu

Audrey N. Tong audrey.tong@nist.gov

Oleg Aulov

oleg.aulov@nist.gov

Ian M. Soboroff

ian.soboroff@nist.gov

Jonathan Washington

jwashin1@swarthmore.edu

Xiaobing Zhao

13522842727@163.com

1 Potamu Research Ltd, Dublin, Ireland

2 Fondazione Bruno Kessler, Trento, Italy

3 National Institute of Standards and Technology, Gaithersburg, MD, USA

4 Swarthmore College, Swarthmore, PA, USA

5 Minzu University of China, Beijing, China 
The paper by Ashengo et al. proposes a context-based MT (CBMT) with RNN approach to build English-Amharic models. Amharic is a morphologically rich language and requires larger parallel corpus to train MT systems to achieve comparable performance. They followed the setup proposed by Niehues et al. (2016) and Zhou et al. (2017) to first use CBMT to translate source text into CBMT target text, which is then combined with the original source text as the input of NMT system. The CBMT system takes advantage of bilingual dictionary, a large target corpus and synonym finder to translate the source into CBMT target text. The resulting NMT model is trained using source and CBMT target as input, and target text as output. The approach alleviates the need of large corpora for MT training. It would be interesting to investigate how it performs in other morphologically rich languages and low-resource languages.

The paper by Sarveswaran et al. presents fundamental work in developing a morphological parser for the Tamil language, ThamizhiMorph. Although the introduction of Byte-Pair Encoding (BPE) to NMT has made training the systems using subwords much more efficient, MT for morphologically rich languages like Tamil is still a challenging task. Tamil exhibits two-level morphology, i.e., a word is represented at two levels: the lexical level and the surface level. The FST-based morphological parser ThamizhiMorph described here performs very well for the Tamil language. We are looking forward to seeing comparisons of NMT systems trained with corpora processed by BPE and ThamizhiMorph.

The paper by Dewangan et al. gives an in-depth review of MT for Indian languages. There are five major language groups spoken in Indian, in which there are 16 major languages. The paper surveys MT systems developed for the two main language groups in India, i.e., Indo-Aryan languages and the Dravidian languages. It also reviews the behaviour of phrases and several linguistic features of six Indian languages and English. Both SMT and NMT were trained with different settings on BPE for seven Indian languages (four of which belong to the Indo-Aryan group and three to the Dravidian group). It is good to have systematic experiments on major Indian languages and we hope to see more research on MT for additional Indian languages in all five Indian language groups.

We are grateful to all the researchers who helped review the second part of the special issue in this challenging time: Alberto Poncelas, Atul Kr. Ojha, Audrey N. Tong, Chen-Tse Tsai, Guodong Xie, Koel Dutta Chowdhury, Oleg Aulov, John Ortega, Juan Alonso, Sangjie Duanzhu, Santanu Pal, and Thepchai Supnithi. Thank you all for making this special issue possible!

Publisher's Note Springer Nature remains neutral with regard to jurisdictional claims in published maps and institutional affiliations. 